Strictly, the conclusions of Nilsson et al. apply only to peptides found in sensory neurones. However, the results with bombesin and vasopressin ${ }^{4,5}$, which have not so far been found in sensory neurones, strongly suggest that other parts of the peripheral nervous system may also exert proliferative influences on normal, stimulated or developing cell populations. One intriguing consequence of these investigations may be to reveal for the first time a defined cellular origin for an identified substance with mitogenic action.

Michael R. Hanley is in the Department of Biochemistry, Imperial College of Science and Technology, London SW7 $2 A Z$, UK.
1. Nilsson, J., Von Euler, A.M. \& Dalsgaard, C.J. Nature 315, 61 (1985).

2. Jessel, T.M. \& Womack, M.D. Trends Neurosci. 8, 43 (1985).

3. Lee, C.M., Iversen, L.L., Hanley, M.R. \& Sandberg, B.E.B. Naunyn-Schmiedeberg's Arch. Pharmac. 318, 281 (1982). Rozengurt, E., Legg, A. \& Pettican, P. Proc. natn. Acad. Sci. U.S.A. 76, 1284 (1979).

Rozengurt, E. \& Sinnet-Smith, J. Proc. natn. Acad. Sci. U.S.A. 80, 2936 (1983).

6. Miller, R.P., Husain, F., Svenssen, M. \& Lohin, S. Endocrinology 100, 1365 (1977).

7. Hunt, N.H., Perris, A.D. \& Sandford, P.A. J. Endocr. 72 , 5 (1977).

8. Willey, J.C., Lechner, J.F. \& Harris, C.C. Expl Cell Res. 153, 245 (1984).

153, 245 (1984).
Hanley, M.R. et al. Nature 309, 258 (1984).

10. Moghimzadeh, F. et al. Neuroscience 10, 553 (1983).

11. Berridge, M.J. Biochem. J. 220, 345 (1984).

12. Buck, S.H. \& Burks, T.F. Trends Pharmac. Sci. 4, 84 (1983). 13. Fujita et al. Expl Eye Res. 38, 165 (1984).

14. Weihe, E., Hartschuh, W. \& Reinecke, E. in Substance $P$. Dublin 1983 (eds Skrabanek, P. \& Powell, D.) 143 (Boole Press, Dublin).

\title{
Protein structure
}

\section{Hands on the calcium switch}

\section{from C. Schutt}

THERE is no such thing as a boring protein; newly solved protein structures always contain surprises. Yet the calmodulin structure published on page 37 of this issue ${ }^{1}$, and the closely similar troponin-C molecule ${ }^{2,3}$, turn out to be even more interesting than usual. The simple 'dumbbell'-shaped molecules, with their easily grasped polypeptide fold, invite close scrutiny of the fundamental mechanism of molecular switching.

These proteins sense intracellular calcium signals and trigger, through changes in conformation, appropriate responses in associated proteins. Calmodulin is a ubiquitious, highly conserved, eukaryotic protein implicated in many coordinated, calcium-dependent cellular responses ${ }^{4}$, whereas troponin- $\mathrm{C}$ is part of a protein complex bound to actin filaments in vertebrate muscle that 'switches on' contraction when calcium is released from the sarcoplasmic reticulum ${ }^{5}$.

The three-dimensional organization of calmodulin is easy to visualize: knobs at both ends of a long exposed helix, with each knob having paired, back-to-back, calcium-binding subdomains (EF-hands) of the type first described for parvalbumin ${ }^{6}$. An EF-hand is a structural motif (see figure) with a 12-residue calcium-binding loop, pictured as the middle finger of the right hand, flanked by two nearly perpendicular helices, represented by thumb and forefinger. In the crystal structure, the concluding helix of the second calciumbinding subdomain runs without interruption into the starting helix of the third binding subdomain, forming the long central helix. It is the splendid isolation of this helix that is unexpected - the tertiary fold of the knobs had been predicted.

In a pioneering use of sequence homology to predict protein structure, troponin- $C$ was determined to have four calcium-binding subdomains ${ }^{7-9}$. Using the criterion of compactness and structural homology with the parvalbumin molecule, Kretsinger and Barry produced a pleasing model of troponin-C with four EFhands symmetrically packed around a hydrophobic core ${ }^{10}$. This model has recently been challenged by Tsalkova and Privalov" naturation results that the protein melts as if comprising three independently folded 'cooperative units'. They conclude that the middle part of the sequence, now
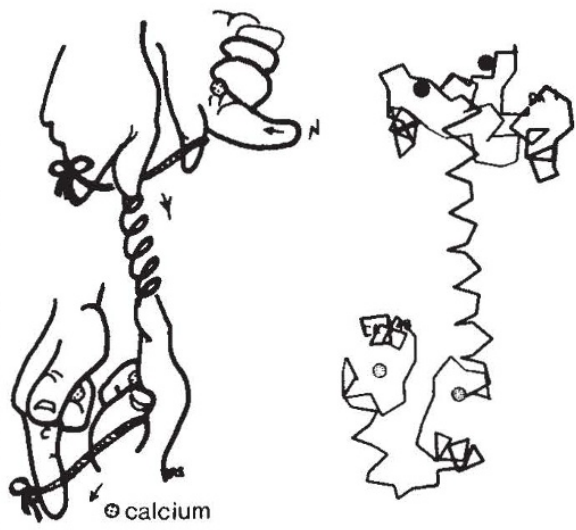

known to form the central helix, somehow prevents the other two units from interacting - a remarkable prediction in view of the three-dimensional structures just published. It is also known that limited proteolysis can split these molecules into two intact but non-associating calcium-binding domains ${ }^{12}$. Would this information have helped to get the prediction right? Perhaps such zero-dimensional experiments will become a more important component in future attempts to predict protein structure from sequence homology.

How might the binding of calcium to such a structure act as a conformational switch? In the case of troponin-C, two of the calcium-binding loops (in the aminoterminal lobe) lack calcium in the crystal and, significantly, have altered inter-helix angles from the standard EF-hand, the ๑) 1985 Nature Publishing Group thumb and forefinger being nearly parallel. These differences are large enough to be recognized by other proteins, especially if there is a relative rotation of the two lobes about the axis of the central helix. A more radical (but not unreasonable) suggestion by Herzberg and James ${ }^{2}$ is that a glycine residue in the middle of the long central helix serves as a pivot about which the two halves of the dumbbell can freely rotate, perhaps even coming into contact. Such a dramatic change could certainly serve as a switch, possibly initiated by ligand-induced changes in the calcium-binding subdomain at the amino-terminus of the helix.

The question, then, is whether the secret of this molecular switch lies with the instability of the long bridging helix? Proteolytic cleavage studies show that this region does have an altered conformation in the absence of calcium. Furthermore, thermal denaturation studies ${ }^{11}$ identify the helix as the least stable part of the structure. Perhaps an exposed helix - a rare feature in protein structures - tottering on the edge of conformational stability has exactly the required sensitivity to respond to energies produced by calcium binding. One is reminded of glucagon, a small protein of borderline stability in solution, which only reveals its helical conformation in the crystalline state ${ }^{13}$. Such a delicate balance between order and disorder could be the basis of switching in all these molecules.

It is tempting to carry these speculations too far. Calmodulin and troponin- $\mathrm{C}$ are only functional while interacting with other proteins and the central helix could well be buried in these situations, or more subtle aspects of protein-protein recognition might predominate. Nevertheless, particularly by the use of genetically engineered mutants, these proteins may yield deep insights into the relationship between the signalling and stability properties of proteins. Finally, we might ask why such a simple structure was not anticipated, when most of its subdomains had been correctly predicted? My guess is that someone, somewhere, did get it right - but who would want to go down as the first to predict a dumbbell when, if the prediction was wrong, their name might so easily become attached to it?

1. Babu, Y.S. et al. Nature 315, 37 (1985).

2. Herzberg, O. \& James, M.N.G. Nature 313, 653 (1985).

3. Sundaralingam, M. et al. Science 227, 945 (1985).

4. Cheung, W.Y. Science 207, 19 (1980).

5. Weber, A. \& Murray, J.M. Physiol. Rev. 53, 612 (1973).

6. Kretsinger, R.H. \& Nockolds, C.E. J. biol. Chem. 248, 313 (1973).

7. Kretsinger, R.H. Nature new Biol. 240, 85 (1972).

8. Collins, J.H., Potter, J.H., Horn, M.J., Wiltshire, G. \& Jackman N. FEBS Lett. 36, 268 (1972).

9. Tufty, R.M. \& Kretsinger, R.H. Science 187, 167 (1975).

10. Kretsinger, R.H. \& Barry, C.D. Biochim. biophys. Acta 405, 40 (1975)

11. Tsalkova, T.N. \& Privalov, P.L. J. molec. Biol. 181, 533 (1985).

12. Newton, D.L., Oldewurtel, M.D., Krinks, M.H., Shiloach, J. \& Klee, C.B. J. biol. Chem. 259, 4419 (1984).

3. Panjipan, B. \& Gratzer, W.B. Eur. J. Biochem. 45, 547 (1974).

C. Schutt is at the Medical Research Council Laboratory of Molecular Biology, Hills Road, Cambridge CB2 $2 Q H$, UK. 